Division of Infectious Diseases, Department of Medicine, Faculty of Medicine and Dentistry, University of Alberta, Edmonton, Canada

2 Department of Pharmacy Practice and Science, University of Maryland School of Pharmacy, Baltimore, Maryland, USA

3 Department of Medicine, Division of Infectious Diseases, UPMC Health System and the University of Pittsburgh School of Medicine, Pittsburgh, PA 15213, USA

Correspondence to: E K McCreary mccrearye3@upmc.edu

Cite this as: BMJ2020;371:m4560 http://dx.doi.org/10.1136/bmj.m4560 Published: 24 November 2020

\section{Remdesivir: a pendulum in a pandemic}

\section{New guidance challenges remdesivir use for patients with covid-19}

\section{Ilan S Schwartz, ${ }^{1}$ Emily L Heil, ${ }^{2}$ Erin K McCreary ${ }^{3}$}

Covid-19 has claimed over a million lives globally and devastated healthcare systems, economies, and societal welfare. Determining effective, safe, and scalable prevention and treatment measures is the top priority of the scientific community. Rapidly disseminating guidance as new data emerge regarding experimental therapies is also of utmost importance.

The World Health Organisation (WHO) living guideline on drugs for covid-19 is updated in The BMJ in response to interim results from the SOLIDARITY trial for repurposed antiviral therapies. ${ }^{1}$ With this version of the guideline, the WHO and partners provide a weak recommendation against the use of remdesivir for patients with any severity of covid-19. This comes after the panel's previous weak recommendation for remdesivir in patients with severe covid-19, which had been made with caveats: that uncertain clinical benefits and cost-effectiveness of the agent may exacerbate existing health inequities, and that, given the uncertainty, active enrolment into ongoing randomised controlled trials should be continued.

Enter SOLIDARITY, a WHO sponsored, multinational, pragmatic, adaptive, open-label trial that randomised hospitalised patients to four repurposed antiviral therapies (hydroxychloroquine, remdesivir, lopinavir/ritonavir, interferon- $\beta 1 \mathrm{a}$ ) or standard care. ${ }^{3}$ The hydroxychloroquine, lopinavir/ritonavir, and interferon- $\beta 1 \mathrm{a}$ arms were discontinued for futility. Among the nearly 5500 patients randomised to remdesivir or standard care, no difference was observed in the primary outcome of in-hospital mortality. Moreover, there were no differences in secondary endpoints including deterioration to require mechanical ventilation or time to discharge.

These data were a disappointment for clinicians who had been encouraged by the first US National Institute of Health sponsored Adaptive Covid-19 Treatment Trial (ACTT-1), a double-blind, placebo controlled trial in which hospitalised patients randomised to a 10 day course of remdesivir had a four day shorter recovery time compared with placebo (median $11 v 15$ days). ${ }^{4}$ Mortality was not significantly different between remdesivir and placebo $(7.1 \% \mathrm{v}$ $11.9 \%$, hazard ratio 0.70 ( $95 \%$ CI 0.47 to 1.04$)$ ), but hope remained that the larger SOLIDARITY trial would demonstrate a mortality benefit. Alas, it did not.

How can the results from ACTT-1 and the SOLIDARITY trial be reconciled? Although the double-blind design of ACTT-1 is typically taken as the gold standard for clinical trials, the open-label, pragmatic nature of SOLIDARITY provides insight into remdesivir's effectiveness in clinical practice across the global community, which is of primary importance in a pandemic. Indeed, findings from the similarly designed (open-label, pragmatic) RECOVERY trial have already changed practice, as academic and clinical communities openly embraced the findings of mortality benefit with dexamethasone. ${ }^{5}$

Universal application of the findings of either study outside the settings in which they were conducted is challenging; for example, the value of reducing time to recovery (thereby potentially reducing hospital length of stay) - a benefit shown convincingly in ACTT-1 but which SOLIDARITY was not optimally designed to evaluate-will vary globally and must be questioned in the context of substantial drug expense.

Finally, the benefits of remdesivir observed in ACTT-1 were seemingly driven by the subgroup of patients requiring only low flow supplemental oxygen at the time of enrolment. This benefit was not seen in the randomised controlled trial by Wang et al, ${ }^{6}$ in which the patient population largely comprised the subgroup demonstrating the greatest benefit in ACTT-1, although that study may have been underpowered. When the results of all four randomised, controlled trials of remdesivir are analysed collectively, we cannot accept the potential benefit of treatment in low risk patients without also acknowledging that remdesivir may cause harm in high risk patients. 3467

Accordingly, the WHO panel noted that the totality of available data does not prove remdesivir is ineffective; rather, there is no evidence that it improves patient-important outcomes that would justify recommending routine use for the average hospitalised patient. WHO support further enrolment into randomised clinical trials to evaluate the role of remdesivir for patients with covid-19, particularly trials in patient populations hypothesised to benefit, such as those needing only low flow oxygen or those with immunocompromising conditions. The many questions that remain regarding remdesivir therapy include the optimal patient population, timing of initiation in relation to symptom onset, duration of therapy, effect on clinical outcomes, and, importantly, the role in combination therapy with corticosteroids, all of which are unknown. ${ }^{8}$

Remdesivir was the best prospect among currently available antiviral therapies for covid-19. However, the equivocal findings to date are not enough to justify widespread use. The WHO's new recommendation is scientifically and economically sound, as widespread use of remdesivir in hospitalised patients is unlikely to save lives, may hinder evaluation of other experimental therapies in clinical trials, and could be economically devastating. 
The impact of these recommendations are likely to be uneven: use of remdesivir in the treatment of covid-19 was already very limited in the vast majority of countries due to supply constraints, high drug costs, and lack of evidence that it reduces mortality. However, there will be an uncomfortable reckoning in countries, such as the US, where remdesivir has become de facto standard care.

Given the complexity of covid-19 and a constantly shifting therapeutic landscape, the incremental benefits of various interventions including remdesivir, immunomodulating agents, ventilation strategies, and anticoagulation are still unknown across the spectrum of illness, and may not be known for years to come. The story of standard care for covid-19 is still being written. In the interim, the WHO guidance appropriately places remdesivir as a potential treatment that needs continued exploration in randomised trials.

Competing interests: The BMJ has judged that there are no disqualifying financial ties to commercial companies. The authors declare the following other interests: EKM has served on advisory boards related to antibacterial agent development for AbbVie, Merck, Shionogi, Summit, and Entasis. ISS and ELH have no interests to disclose. The BMJ policy on financial interests is here:

https:/www.bmj.com/sites/default/files/attachments/resources/2016/03/16-current-bmj-educationcoi-form.pdf.

1 Rochwerg B, Agoritsas T, Lamontagne F, etal. A living WHO guideline on drugs for covid-19 [Update 1]. BM/2020;370:m3379. doi: 10.1136/bmj.m3379 pmid: 32887691

2 Rochwerg B, Agarwal A, Zeng L, etal. Remdesivir for severe covid-19: a clinical practice guideline. BMJ2020;370:m2924. doi: 10.1136/bmj.m2924 pmid: 32732352

3 Pan H, Peto R, Karim QA, etal. Repurposed antiviral drugs for COVID-19- interim WHO SOLIDARITY trial results. medRxiv 2020; doi: 10.1101/2020.10.15.20209817.

4 Beigel JH, Tomashek KM, Dodd LE, etalACTT-1 Study Group Members. Remdesivir for the treatment of Covid-19 - final report. N Engl J Med 2020;383:1813-26. doi: 10.1056/NEJMoa2007764 pmid: 32445440

5 Horby P, Lim WS, Emberson JR, etalRECOVERY Collaborative Group. Dexamethasone in hospitalized patients with Covid-19 - preliminary report. N Engl J Med 2020 doi: 10.1056/NEJMoa2021436. pmid: 32678530

6 Wang Y, Zhang D, Du G, etal. Remdesivir in adults with severe COVID-19: a randomised double-blind, placebo-controlled, multicentre trial. Lancet 2020;395:1569-78. doi: 10.1016/S0140-6736(20)31022-9 pmid: 32423584

7 Spinner CD, Gottlieb RL, Criner GJ, etalGS-US-540-5774 Investigators. Effect of remdesivir vs standard care on clinical status at 11 days in patients with moderate COVID-19: a randomized clinical trial. JAMA 2020;324:1048-57. doi: 10.1001/jama.2020.16349 pmid: 32821939

8 McCreary EK, Angus DC. Efficacy of remdesivir in COVID-19. JAMA 2020;324:1041-2 doi: 10.1001/jama.2020.16337 pmid: 32821934

This article is made freely available for use in accordance with BMJ's website terms and conditions for the duration of the covid-19 pandemic or until otherwise determined by BMJ. You may use, download and print the article for any lawful, non-commercial purpose (including text and data mining) provided that all copyright notices and trade marks are retained. 